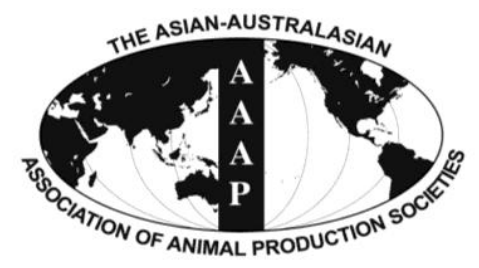

Asian Australas. J. Anim. Sci.

Vol. 26, No. 11 : 1536-1544 November 2013

http://dx.doi.org/10.5713/ajas.2013.13356

www.ajas.info

pISSN 1011-2367 elSSN 1976-5517

\title{
A Least Squares Regression Model to Detect Quantitative Trait Loci with Polar Overdominance in a Cross of Outbred Breeds: Simulation
}

\author{
Jong-Joo Kim* and Jack C. M. Dekkers ${ }^{1}$ \\ School of Biotechnology, Yeungnam University, Gyeongsan, Gyeongbuk, Korea
}

\begin{abstract}
A least squares regression interval mapping model was derived to detect quantitative trait loci (QTL) with a unique mode of genomic imprinting, polar overdominance (POD), under a breed cross design model in outbred mammals. Tests to differentiate POD QTL from Mendelian, paternal or maternal expression QTL were also developed. To evaluate the power of the POD models and to determine the ability to differentiate POD from non-POD QTL, phenotypic data, marker data and a biallelic QTL were simulated on 512 $\mathrm{F}_{2}$ offspring. When tests for Mendelian versus parent-of-origin expression were performed, most POD QTL were classified as partially imprinted QTL. The application of the series of POD tests showed that more than $90 \%$ and $80 \%$ of medium and small POD QTL were declared as POD type. However, when breed-origin alleles were segregating in the grand parental breeds, the proportion of declared POD QTL decreased, which was more pronounced in a mating design with a small number of parents $\left(\mathrm{F}_{0}\right.$ and $\left.\mathrm{F}_{1}\right)$. Non-POD QTL, i.e. with Mendelian or parent-of-origin expression (complete imprinting) inheritance, were well classified (>90\%) as non-POD QTL, except for QTL with small effects and paternal or maternal expression in the design with a small number of parents, for which spurious POD QTL were declared. (Key Words: QTL, Imprinting, Polar Overdominance, Pig, Breed-cross)
\end{abstract}

\section{INTRODUCTION}

Genomic imprinting, a non-Mendelian form of gene expression, has been one of the interesting research areas in mammalian genetics due to its unique expression and inheritance mechanisms and its important role in growth, development, and behavior (Tycko and Morison, 2002; Wilkens and Haig, 2003). One typical imprinted gene, insulin-like growth factor 2 (IGF2), has been shown to influence body composition in swine (Jeon et al., 1999; Nezer et al., 1999). Genomic regions with imprinted effects can be detected using QTL interval mapping in $\mathrm{F}_{2}$ crosses between lines or breeds that segregate for marker alleles because of the ability to follow parental origin (Thomsen et al., 2004; Choi et al., 2011; Li et al., 2011). Development and application of statistical methods to detect chromosomal regions or quantitative trait loci (QTL) with imprinting effects in a breed cross design have been developed and implemented for $\mathrm{F}_{2}$ crosses of breeds of

\footnotetext{
* Corresponding Author: Jong-Joo Kim. Tel: +82-53-810-3027, Fax: +82-53-801-3027, E-mail: kimjj@ynu.ac.kr

${ }^{1}$ Department of Animal Science, Center for Integrated Animal Genomics, Iowa State University, Ames, IA 50011, USA.

Submitted Jun. 25, 2013; Accepted Aug. 19, 2013; Revised Aug. 28, 2013
}

swine (Knott et al., 1998; de Koning et al., 2000; Ruckert et al., 2012). A specific mode of genomic imprinting that has been uncovered in mammals is polar overdominance (POD), for which one type of heterozygous progeny differs in phenotype from the other heterozygous type, depending on the parent from which the mutant allele was received, and also differs from the two types of homozygotes. The only known phenotype caused by this unique expression in mammals is the 'callipyge' phenotype, which is expressed as muscular hypertrophy in sheep (Cockett et al., 1996). Subsequent evaluations found that the lambs with the callipyge-causing genotype at the callipyge locus $(C L P G)$ had heavier carcass, greater fat-free lean, and less fat than those with the other genotypes (Freking et al., 1998). In swine, a $D L K$ polymorphism in the CLPG gene was also reported to be associated with growth and fatness in a cross of commercial pig breeds (Kim et al., 2004).

The first objective of this study was to derive a POD model for a genome-scan in a cross between outbred breeds or lines, so as to enable a systematic search of other regions or loci that exhibit POD across the genomes of outbred animals. The second was to validate the proposed POD models by performing simulation studies with different mapping designs and sample sizes, and different modes of inheritance, QTL effects, and allele frequencies in parental 
breeds.

\section{MATERIALS AND METHODS}

\section{Polar overdominance QTL models}

Derivation of the model was based on the framework of the least-squares regression interval mapping method for detection of QTL in a breed-cross model, which detects differences in the average effects of alleles that originated from the two parental breeds (Haley et al., 1994). There are four possible QTL genotypes among $\mathrm{F}_{2}$ animals in the breed-cross model based on breed origin, 11, 12, 21, 22, (1 and 2 represent alternate QTL alleles derived from parental breeds 1 and 2, and the first allele represents the allele received from the $F_{1}$ sire). Following de Koning et al., (2002), the full imprinting model for a putative QTL at a given position is:

$$
\mathrm{Y}=\mathrm{Xb}+\mathrm{a}_{\mathrm{pat}} \mathrm{P}_{\mathrm{pat}}+\mathrm{a}_{\mathrm{mat}} \mathrm{P}_{\mathrm{mat}}+\mathrm{dP}_{\mathrm{d}}+\mathrm{e}
$$

Where, $\mathrm{Y}$ is a vector of phenotypes on $\mathrm{F}_{2}$ animals, $\mathrm{X}$ is a design matrix, $b$ is a vector of fixed and covariate effects, and $\mathrm{e}$ is a vector of uncorrelated residuals with constant variance. The coefficients $a_{\text {pat }}$ and $a_{\text {mat }}$ are the effects associated with the paternally and maternally inherited breed specific QTL alleles, respectively, and $\mathrm{P}_{\text {pat }}$ and $\mathrm{P}_{\text {mat }}$ are the corresponding vectors with probabilities for each animal at the putative QTL position conditional on flanking marker genotypes, such that $\mathrm{P}_{\text {pat }}=\left(\mathrm{P}_{11}+\mathrm{P}_{12}\right)-\left(\mathrm{P}_{21}+\mathrm{P}_{22}\right)$ and $\mathrm{P}_{\text {mat }}=\left(\mathrm{P}_{11}+\mathrm{P}_{21}\right)-\left(\mathrm{P}_{12}+\mathrm{P}_{22}\right)$, where $\mathrm{P}_{\mathrm{ij}}$ is the vector of probabilities of inheriting QTL genotype ij. The effects of the four possible QTL genotypes in terms of parameters of the full model (1) are $\left(a_{\text {pat }}+a_{\text {mat }}\right),\left(a_{\text {pat }}-a_{\text {mat }}+d\right),\left(-a_{\text {pat }}+a_{\text {mat }}+d\right)$, and $\left(-a_{\text {pat }}-a_{\text {mat }}\right)$, respectively (Figure 1$)$. To generate POD models, restrictions were put on the QTL genotype effects, such that in a type I POD model (here we refer to type I and type II POD when the differential phenotype is observed for QTL genotype 12 and for genotype 21, respectively), genotype effects of 11,21 , and 22 are the same, such that $\left(\mathrm{a}_{\text {pat }}+\mathrm{a}_{\text {mat }}\right)=\left(-\mathrm{a}_{\text {pat }}+\mathrm{a}_{\text {mat }}+\mathrm{d}\right)$ and $\left(-\mathrm{a}_{\text {pat }}+\mathrm{a}_{\text {mat }}+\mathrm{d}\right)=\left(-\mathrm{a}_{\text {pat }}-\mathrm{a}_{\text {mat }}\right)$. This makes $a_{\text {pat }}=1 / 2 d$ and $a_{\text {mat }}=-1 / 2 d$, where $d$ represents the dominance effect of the 12 genotype over the average of the three other genotypes. Using this parameterization in model 1, the type I POD model becomes:

$$
\mathrm{Y}=\mathrm{Xb}+\mathrm{d}\left(1 / 2 \mathrm{P}_{\mathrm{pat}}-1 / 2 \mathrm{P}_{\mathrm{mat}}+\mathrm{P}_{\mathrm{d}}\right)+\mathrm{e}
$$

which reduces to:

$$
\mathrm{Y}=\mathrm{Xb}+2 \mathrm{dP}_{12}+\mathrm{e}
$$

Similarly, the type II POD model is:

$$
\mathrm{Y}=\mathrm{Xb}+2 \mathrm{dP}_{21}+\mathrm{e}
$$

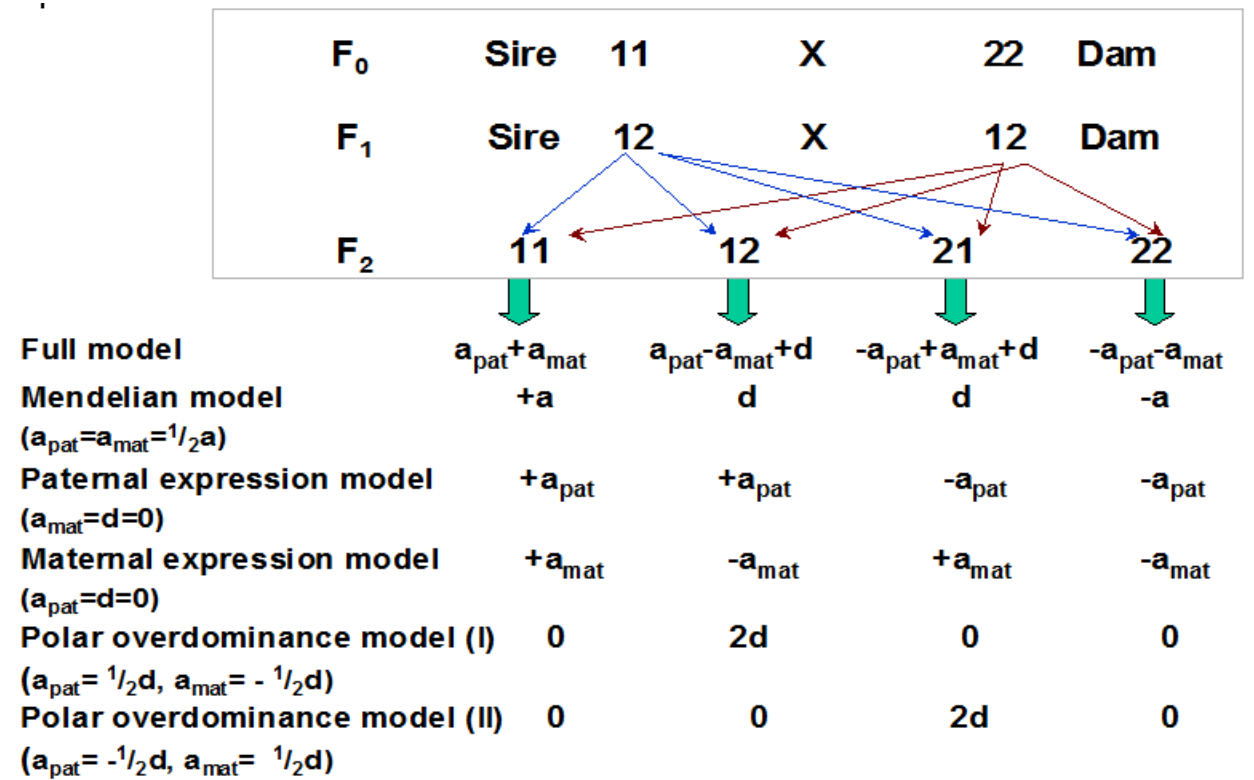

Figure 1. Models and coefficients for QTL effects for each genotype in a cross between two outbred breeds or lines with alternate QTL alleles ( 1 and 2$)$ in a three generation breed cross population. In the full model, both homozygotes have different additive effects depending on inheritance of alternate alleles through the $\mathrm{F}_{1}$ sire $\left(\mathrm{a}_{\mathrm{pat}}\right)$ and $\mathrm{F}_{1}$ dam $\left(\mathrm{a}_{\mathrm{mat}}\right)$. Heterozygotes have an additional dominance effect (d). Restrictions (in parentheses) were imposed on QTL effects in the full model (De Koning et al., 2002) to identify: Mendelian QTL when there is no difference of additive effects between alleles derived through the $\mathrm{F}_{1}$ sire and $\mathrm{F}_{1}$ dam; paternally (maternally) expressed QTL, when alternate alleles inherited through $\mathrm{F}_{1}$ sire (dam) differ in effect; type I (II) polar overdominance QTL, when the differential phenotype is observed for QTL genotype 12 (21). 
Parameterizations to derive Mendelian, paternal, and maternal expression models are in Figure 1, following de Koning et al. (2002), resulting in:

$$
\begin{aligned}
& Y=X b+a P_{a}+d P_{d}+e \\
& Y=X b+a_{\text {pat }} P_{\text {pat }}+e \\
& Y=X b+a_{\text {mat }} P_{\text {mat }}+e
\end{aligned}
$$

Where $\mathrm{a}$ is the additive QTL effect and $\mathrm{P}_{\mathrm{a}}$ is a vector of probabilities $\left(\mathrm{P}_{11}-\mathrm{P}_{22}\right)$, and the other variables are as in (1).

To estimate genetic variance due to a gene with POD effect, the population mean at the QTL under random mating was derived with parameters $p$ and $q$ (alternate allele frequencies from the two parental breeds), and $d$ (dominance effect as in the above POD model). If a gene has complete POD inheritance mode, then one heterozygote has a genotype effect of $2 d$ and the other three genotypes have no additive $(a)$ or dominance effect. The population mean then becomes $2 d p q$ and the genetic variance due to the POD QTL is $4 p q d^{2}(1-p q)$. Under random mating, breed-origin allele frequencies, $p$ and $q$ are equal to 0.5 in the $\mathrm{F}_{2} \mathrm{~S}$ of a breed-cross population, such that the mean and variance become $0.5 d$ and $0.75 d^{2}$. For Mendelian and complete imprinting QTL, genetic variances are $2 p q[a+$ $d(q-p)]^{2}+(2 p q d)^{2}$ and $4 p q a^{2}$, respectively (de Koning et al., 2002). In an $F_{2}$ population where breed-origin QTL alleles have equal frequency $(p=q=0.5)$, the variances become $0.5 a^{2}+0.25 d^{2}$ and $a^{2}$, respectively.

To detect POD QTL, an $F$-test statistic for models 2 and 3 against the null hypothesis of no QTL $\left(F_{P O D}\right)$ can be obtained at unit increments along the chromosome (Test 1). For significant QTL based on Test 1, the following two sets of tests were performed to determine whether the POD model fits better than the full, Mendelian, paternal, or maternal expression models (Tests 2 and 3 ).

Test 2. $F_{L O F}: \mathrm{Ha}=$ full imprinting model (1) vs $\mathrm{Ho}=$ POD model ( 2 or 3 ). This test was conducted at the most likely position under the POD model to determine whether the POD model does not have a significantly poorer fit than the full imprinting model. Test statistic $F_{L O F}$ has an $F$ distribution with 2 and $n-4$ ( $n$ is the number of $F_{2} \mathrm{~s}$ ) degrees of freedom.

Test 3. Ha = POD model (2 or 3) vs Ho = Mendelian (4), paternal (5), and maternal (6) expression models, using the following likelihood ratio test statistics, respectively:

$$
\begin{aligned}
& \mathrm{LRT}_{\mathrm{MEND}}=n * \ln \left(\mathrm{RSS}_{\mathrm{MEND}} / \mathrm{RSS}_{\mathrm{POD}}\right), \\
& \mathrm{LRT}_{\mathrm{PAT}}=n * \ln \left(\mathrm{RSS}_{\mathrm{PAT}} / \mathrm{RSS}_{\mathrm{POD}}\right),
\end{aligned}
$$

$$
\mathrm{LRT}_{\mathrm{MAT}}=n * \ln \left(\mathrm{RSS}_{\mathrm{MAT}} / \mathrm{RSS}_{\mathrm{POD}}\right)
$$

Where, RSS is residual sum of squares of the corresponding model at the most likely position under the POD model, and $n$ is the number of individuals in the $\mathrm{F}_{2}$. Since these are not tests between nested models, significance thresholds were derived empirically by randomly switching elements of $\mathrm{P}$ vectors (within individual); $\mathrm{P}_{11}$ vs $\mathrm{P}_{12}$ for $\mathrm{LRT}_{\mathrm{PAT}}$ and $\mathrm{P}_{12}$ vs $\mathrm{P}_{22}$ for $\mathrm{LRT}_{\mathrm{MAT}}$ in the type I POD model; $\mathrm{P}_{21}$ vs $\mathrm{P}_{22}$ for $\mathrm{LRT}_{\mathrm{PAT}}$ and $\mathrm{P}_{11}$ vs $\mathrm{P}_{21}$ for LRT $_{\mathrm{MAT}}$ in the type II POD model; and $\mathrm{P}_{12}$ vs $\mathrm{P}_{21}$ for LRT $_{\text {MEND }}$ in both models. A QTL with POD mode of gene action was declared when Ho wa not rejected in Test 2 and all Ho's were rejected in Test 3.

\section{Mendelian vs parent-of-origin expression analysis}

POD QTL were tested with a series of tests to differentiate parent-of-origin effects (complete imprinting) from Mendelian effects. $F$-tests between Mendelian and parent-of-origin models were applied according to the decision tree described in Thomsen et al. (2004), with some minor modifications of the specific tests conducted. First, the Mendelian model (4) was tested against the null model. If significant at a 5\% chromosome-wise (CW) level, the full model (1) was tested against the Mendelian model at the best position of the full model within the QTL region. If not significant at the 5\% CW level, a Mendelian QTL was declared, otherwise a QTL with parent-of-origin effects. When the Mendelian model was not significant in the first step, the full model was tested against the null model and a QTL with parent-of-origin effects was declared if significant at the 5\% CW level. To determine the nature of the detected parent-of-origin effects, tests of the full against the paternal (5) and maternal (6) models were conducted at the best positions of these respective models within the QTL region. If the full model explained significantly more variation than the paternal model (at the $5 \% \mathrm{CW}$ level) and the full model did not explain more variation than the maternal model $(5 \% \mathrm{CW})$, then $a_{(\mathrm{pat})}=0$ and a maternally expressed QTL was inferred. A paternally expressed QTL was inferred vice versa $\left(a_{(\mathrm{mat})}=0\right)$. If tests of the full against the paternal and maternal expression models were both significant or both not significant, partial expression was inferred (Thomsen et al., 2004). Chromosome-wise thresholds for the tests between alternate expression models were set equal to thresholds obtained for tests against the null hypothesis of no QTL with equivalent degrees of freedom in the numerator of $F$-test statistic, as suggested by Thomsen et al. (2004). Thus, thresholds for tests of the paternal against the null model were used for tests of the full against the Mendelian model, and thresholds for tests of the Mendelian against the null model were used to test the full model against the paternal and maternal models. 


\section{Simulation}

To validate the POD models and tests and to evaluate their power and ability to distinguish alternative QTL types, $\mathrm{F}_{2}$ populations were simulated based on two designs that are relevant to experimental pig QTL mapping populations (Bidanel and Rothschild, 2002). Design I mimicked the Berkshire and Yorkshire $\mathrm{F}_{2}$ cross described in Malek et al. (2001). This design was based on a limited number of parents, with two $\mathrm{F}_{0}$ grand sires of one breed and ten $\mathrm{F}_{0}$ grand dams of another breed, to generate $10 \mathrm{~F}_{1}$ 's per dam. Eight $F_{1}$ sires and $32 F_{1}$ dams were randomly chosen to produce $16 \mathrm{~F}_{2}$ 's per $\mathrm{F}_{1}$ dam, for a total of $512 \mathrm{~F}_{2}$ offspring. The second design was based on a larger number of parents, with $20 \mathrm{~F}_{0}$ sires and $80 \mathrm{~F}_{0}$ dams, with five offspring per dam. A total of $19 \mathrm{~F}_{1}$ sires and $57 \mathrm{~F}_{1}$ dams were randomly chosen to produce $513 \mathrm{~F}_{2}$ progeny (9 per full-sib family). A chromosome of $100 \mathrm{cM}$ was simulated with 11 markers at $10 \mathrm{cM}$ intervals. Markers were simulated with four alleles with frequencies of $0.6,0.2,0.1,0.1$ in one breed and 0.1 , $0.1,0.2,0.6$ in the other breed, following simulations that were designed to mimic the marker information content of the Berkshire and Yorkshire cross (Malek et al., 2001). Biallelic QTL with different modes of inheritance pattern were simulated at position $75 \mathrm{cM}$ on the chromosome: additive, complete dominance, paternal and maternal expression, and type I and II POD effects. QTL effects were defined as large, medium or small QTL, such that the QTL explained $32 \%, 12.5 \%$, or $5.1 \%$, respectively, of the phenotypic variance for the quantitative trait that was simulated. Different QTL effects were set depending on inheritance pattern and defined QTL size, such that the defined QTL sizes were consistent across QTL types (Table 1). Alternate QTL alleles in parental breeds were assumed to be fixed $(1.0 / 0.0)$ or to be distributed with different frequencies $(0.8 / 0.2)$. Five hundred replicate data sets were simulated for each set. Thresholds at the $5 \% \mathrm{CW}$ level for QTL detection in each QTL model were derived from three thousand replicates with QTL effects set to zero (Test 1). Significance thresholds for Tests 2 and 3 were determined at a comparison-wise 5\% level. p Values for Test 2 were obtained using standard $F$ statistic thresholds. Significance threshold values for LRTs (Test 3) to test QTL with POD inheritance versus Mendelian, paternal, and maternal inheritance were obtained with 2000 permutations for each replicate.

\section{RESULTS}

\section{Detection of POD QTL}

Table 2 presents power to detect POD QTL under different genetic models, estimates of QTL position and effects, and of the proportion of phenotypic variance explained by POD QTL under different mapping designs, QTL types and sizes, and allele frequencies in parental breeds. Most POD QTL were detected in their corresponding POD models when alternate alleles were fixed in parental breeds, for all QTL sizes, i.e. power to detect QTL was close to $100 \%$. Also, POD QTL with large or medium size was detected in imprinting models at similar detection rates as in the POD models. However, when QTL alleles were segregating in the parental breeds, power to detect a POD QTL using their respective POD models decreased, especially for small QTL in design I, where a small number of parental animals were used. When increasing the number of $F_{2}$ animals (double the $F_{2}$ size), more POD QTL were detected using their respective POD models when the allele segregated in the parental breeds, e.g. more than $10 \%$ increase for small QTL.

The QTL that were detected with statistical significance under POD models were further classified as POD QTL, using the tests (2 and 3 ) described above. Generally, POD QTL were most often declared to be POD QTL when parental breeds were fixed for alternate alleles, e.g. more than $95 \%$ for large QTL, an around $90 \%$ and $80 \%$ for medium and small QTL, respectively. When QTL size was small, around $10 \%$ of POD QTL were declared to be type I or II (Table 2). When alleles were segregating in parental breeds, the proportion of declared POD QTL decreased substantially across POD QTL types and sizes, and mating designs. The degree of failure to declare a POD QTL was larger for design I than for design II, for large or medium QTL. When increasing the number of $F_{2}$ animals, a marginal increase in frequency of declared POD QTL was observed, especially for the small QTL in design II (Table 2).

Table 1. QTL effect and variance for different QTL sizes and inheritance patterns in the simulation study

\begin{tabular}{lcccc}
\hline \multirow{2}{*}{ QTL inheritance mode } & Genetic variance & \multicolumn{3}{c}{ QTL effect $^{\mathrm{a}}$} \\
\cline { 3 - 5 } & & Large & Medium & Small \\
\hline Additive $(a)$ & $0.5 a^{2}$ & 0.800 & 0.500 & 0.320 \\
Complete dominance $(a=d)$ & $0.5 a^{2}+0.25 d^{2}$ & 0.654 & 0.408 & 0.261 \\
Parental-origin expression $(a)$ & $a^{2}$ & 0.566 & 0.354 & 0.226 \\
Polar overdominance $(d)$ & $0.75 d^{2}$ & 0.654 & 0.408 & 0.261 \\
\hline
\end{tabular}

${ }^{\mathrm{a}}$ Different QTL effects under varying genetic models were set such that large, medium and small QTL explained 32\%, $12.5 \%$ or 5.1\%, respectively, of the phenotypic variance. Error variances were set $0.680,0.875$ and 0.949 for large, medium and small QTL, respectively, such that overall phenotypic variances become standard unit (1.0). 
Table 2. Power to detect polar overdominance (POD) QTL under different genetic models, estimates and standard deviations (in bracket) of POD QTL effects and positions, and proportion of phenotypic variance explained by POD QTL based on simulated data

\begin{tabular}{|c|c|c|c|c|c|c|c|c|c|c|c|c|c|c|c|}
\hline \multirow{2}{*}{$\begin{array}{l}\text { POD QTL effect } \\
\text { (allele } \\
\text { frequency) }{ }^{\mathrm{a}}\end{array}$} & \multicolumn{5}{|c|}{$\begin{array}{l}\text { Power to detect QTL } \\
(\%)^{\mathrm{b}}\end{array}$} & \multicolumn{3}{|c|}{$\begin{array}{l}\text { Declared POD type } \\
(\%)^{\mathrm{c}}\end{array}$} & \multicolumn{4}{|c|}{$\begin{array}{c}\text { Declared non-POD } \\
\text { type }(\%)^{\mathrm{d}}\end{array}$} & \multirow{2}{*}{$\begin{array}{c}\text { POD QTL } \\
\text { position } \\
(\text { true }=75 \mathrm{cM})^{\mathrm{e}}\end{array}$} & \multirow{2}{*}{$\begin{array}{l}\text { POD QTL } \\
\text { effect }(d)^{\mathrm{f}}\end{array}$} & \multirow{2}{*}{$\begin{array}{c}\text { POD QTL } \\
\text { variance }(\%)^{\mathrm{g}}\end{array}$} \\
\hline & Mend & $\begin{array}{l}\text { Pat } \\
\text { Exp }\end{array}$ & $\begin{array}{l}\text { Mat } \\
\text { Exp }\end{array}$ & $\begin{array}{c}\text { Pod } \\
\text { I }\end{array}$ & $\begin{array}{c}\text { Pod } \\
\text { II }\end{array}$ & I & II & both & Mend & $\begin{array}{l}\text { Pat } \\
\text { Exp }\end{array}$ & $\begin{array}{l}\text { Mat } \\
\text { Exp }\end{array}$ & $\begin{array}{l}\text { Partial } \\
\text { Exp }\end{array}$ & & & \\
\hline \multicolumn{16}{|c|}{ Design I $(\mathrm{N}=512)$} \\
\hline I $0.65(1.0)$ & 100 & 100 & 100 & 100 & 100 & 99 & 0 & 0 & 0 & 0 & 0 & 100 & $75.0(1.6)$ & $0.65(0.05)$ & $32.2(5.0)$ \\
\hline I $0.65(0.8)$ & 63 & 79 & 77 & 90 & 82 & 59 & 3 & 6 & 2 & 10 & 8 & 69 & $74.2(9.2)$ & $0.39(0.14)$ & $13.1(8.4)$ \\
\hline I $0.41(1.0)$ & 90 & 97 & 98 & 100 & 76 & 93 & 0 & 6 & 0 & 4 & 4 & 92 & $75.0(3.4)$ & $0.41(0.05)$ & $12.6(3.2)$ \\
\hline I $0.41(0.8)$ & 36 & 55 & 57 & 76 & 48 & 54 & 4 & 10 & 3 & 10 & 12 & 50 & $73.3(11.9)$ & $0.27(0.09)$ & $6.1(3.3)$ \\
\hline I $0.26(1.0)$ & 42 & 63 & 66 & 99 & 37 & 83 & 1 & 12 & 7 & 17 & 17 & 55 & $74.8(7.1)$ & $0.27(0.05)$ & $5.5(2.2)$ \\
\hline I $0.26(0.8)$ & 16 & 30 & 33 & 56 & 26 & 41 & 4 & 9 & 7 & 6 & 9 & 28 & $72.6(15.6)$ & $0.21(0.07)$ & $3.6(1.6)$ \\
\hline II $0.65(1.0)$ & 100 & 100 & 100 & 100 & 100 & 0 & 98 & 1 & 0 & 0 & 0 & 100 & 74.9 (1.6) & $0.65(0.05)$ & $31.9(4.8)$ \\
\hline II $0.65(0.8)$ & 62 & 76 & 79 & 79 & 90 & 2 & 59 & 8 & 3 & 9 & 11 & 67 & $74.5(8.5)$ & $0.38(0.15)$ & $12.4(8.8)$ \\
\hline II $0.41(1.0)$ & 91 & 97 & 97 & 79 & 100 & 0 & 89 & 10 & 0 & 2 & 3 & 95 & $74.9(3.3)$ & $0.41(0.05)$ & $12.9(3.2)$ \\
\hline II $0.41(0.8)$ & 34 & 58 & 55 & 50 & 79 & 5 & 57 & 11 & 3 & 12 & 10 & 50 & $74.7(9.8)$ & $0.27(0.09)$ & $6.2(3.7)$ \\
\hline II $0.26(1.0)$ & 43 & 68 & 66 & 42 & 99 & 1 & 81 & 16 & 3 & 17 & 18 & 59 & $74.3(7.2)$ & $0.27(0.05)$ & $5.7(2.0)$ \\
\hline II $0.26(0.8)$ & 16 & 36 & 26 & 26 & 57 & 6 & 42 & 7 & 5 & 8 & 5 & 30 & $73.3(14.9)$ & $0.21(0.07)$ & $3.7(1.8)$ \\
\hline \multicolumn{16}{|c|}{ Design $I(N=1024)$} \\
\hline I $0.41(1.0)$ & 99 & 100 & 100 & 100 & 97 & 93 & 0 & 2 & 0 & 0 & 0 & 100 & $75.0(1.9)$ & $0.41(0.04)$ & $12.6(2.4)$ \\
\hline I $0.41(0.8)$ & 53 & 76 & 72 & 88 & 75 & 51 & 4 & 6 & 2 & 11 & 7 & 69 & $74.2(9.2)$ & $0.25(0.09)$ & $5.2(3.2)$ \\
\hline I $0.26(1.0)$ & 81 & 94 & 92 & 100 & 68 & 88 & 0 & 8 & 0 & 4 & 6 & 89 & $74.9(4.1)$ & $0.26(0.04)$ & $5.3(1.5)$ \\
\hline I $0.26(0.8)$ & 24 & 45 & 51 & 69 & 44 & 46 & 7 & 6 & 4 & 10 & 13 & 40 & $74.4(10.3)$ & $0.18(0.05)$ & $2.6(1.4)$ \\
\hline II $0.41(1.0)$ & 100 & 100 & 100 & 96 & 100 & 0 & 91 & 2 & 0 & 1 & 0 & 99 & $74.9(2.0)$ & $0.41(0.04)$ & $12.6(2.3)$ \\
\hline II $0.41(0.8)$ & 50 & 71 & 74 & 68 & 86 & 3 & 52 & 4 & 2 & 9 & 12 & 63 & $74.1(8.1)$ & $0.25(0.09)$ & $5.2(3.4)$ \\
\hline II $0.26(1.0)$ & 81 & 95 & 90 & 68 & 100 & 0 & 90 & 7 & 0 & 6 & 7 & 87 & $75.1(4.3)$ & $0.26(0.04)$ & $5.3(1.4)$ \\
\hline II $0.26(0.8)$ & 26 & 53 & 53 & 44 & 73 & 4 & 52 & 7 & 5 & 12 & 12 & 45 & $72.5(12.0)$ & $0.18(0.05)$ & $2.7(1.5)$ \\
\hline \multicolumn{16}{|c|}{ Design II $(\mathrm{N}=513)$} \\
\hline I $0.65(1.0)$ & 100 & 100 & 100 & 100 & 100 & 95 & 0 & 1 & 0 & 0 & 0 & 100 & $74.9(1.5)$ & $0.65(0.05)$ & $32.0(4.5)$ \\
\hline I $0.65(0.8)$ & 47 & 88 & 88 & 99 & 88 & 65 & 3 & 6 & 0 & 9 & 10 & 79 & $74.7(6.1)$ & $0.35(0.09)$ & $9.8(4.9)$ \\
\hline I $0.41(1.0)$ & 87 & 98 & 97 & 100 & 79 & 92 & 1 & 5 & 0 & 3 & 4 & 93 & $75.1(3.5)$ & $0.41(0.06)$ & $12.6(3.4)$ \\
\hline I $0.41(0.8)$ & 20 & 56 & 61 & 90 & 48 & 63 & 4 & 10 & 2 & 12 & 17 & 52 & $74.0(10.3)$ & $0.24(0.06)$ & $4.5(2.2)$ \\
\hline I $0.26(1.0)$ & 47 & 64 & 66 & 99 & 36 & 84 & 0 & 9 & 6 & 15 & 17 & 58 & $74.3(6.6)$ & $0.27(0.05)$ & $5.5(2.0)$ \\
\hline I $0.26(0.8)$ & 10 & 22 & 30 & 51 & 24 & 35 & 8 & 5 & 6 & 6 & 5 & 22 & $72.8(16.4)$ & $0.19(0.04)$ & $2.9(1.1)$ \\
\hline II 0.65 (1.0) & 100 & 100 & 100 & 100 & 100 & 0 & 94 & 1 & 0 & 0 & 0 & 100 & $75.0(1.4)$ & $0.65(0.05)$ & $32.2(4.6)$ \\
\hline II $0.65(0.8)$ & 45 & 90 & 93 & 88 & 99 & 2 & 67 & 5 & 0 & 6 & 10 & 83 & 74.7 (5.7) & $0.35(0.09)$ & $10.0(4.9)$ \\
\hline II $0.41(1.0)$ & 90 & 97 & 97 & 75 & 100 & 0 & 92 & 4 & 0 & 2 & 2 & 96 & $75.1(3.6)$ & $0.41(0.05)$ & $12.7(3.2)$ \\
\hline II $0.41(0.8)$ & 20 & 57 & 61 & 48 & 86 & 4 & 63 & 10 & 3 & 12 & 14 & 49 & $74.2(10.1)$ & $0.24(0.06)$ & $4.5(2.2)$ \\
\hline II $0.26(1.0)$ & 40 & 65 & 69 & 43 & 99 & 0 & 82 & 11 & 4 & 17 & 19 & 57 & $75.0(6.7)$ & $0.27(0.05)$ & $5.6(2.0)$ \\
\hline II $0.26(0.8)$ & 9 & 30 & 27 & 23 & 55 & 8 & 37 & 6 & 5 & 6 & 5 & 29 & $72.0(16.8)$ & $0.19(0.05)$ & $2.9(1.1)$ \\
\hline \multicolumn{16}{|c|}{ Design II $(\mathrm{N}=1026)$} \\
\hline I $0.41(1.0)$ & 100 & 100 & 100 & 100 & 97 & 94 & 0 & 2 & 0 & 0 & 0 & 100 & $74.9(1.8)$ & $0.41(0.04)$ & $12.6(2.2)$ \\
\hline I $0.41(0.8)$ & 41 & 84 & 82 & 96 & 81 & 65 & 1 & 9 & 1 & 13 & 12 & 71 & $74.8(5.6)$ & $0.22(0.05)$ & $4.0(1.9)$ \\
\hline I $0.26(1.0)$ & 79 & 92 & 91 & 100 & 69 & 86 & 0 & 8 & 0 & 7 & 8 & 85 & $75.3(3.8)$ & $0.26(0.04)$ & $5.2(1.6)$ \\
\hline I $0.26(0.8)$ & 19 & 53 & 53 & 84 & 39 & 60 & 5 & 7 & 4 & 14 & 15 & 42 & $74.1(9.8)$ & $0.16(0.04)$ & $2.1(0.9)$ \\
\hline II $0.41(1.0)$ & 100 & 100 & 100 & 97 & 100 & 0 & 96 & 1 & 0 & 0 & 0 & 100 & $75.1(1.6)$ & $0.41(0.04)$ & $12.6(2.3)$ \\
\hline II $0.41(0.8)$ & 36 & 80 & 85 & 78 & 97 & 3 & 68 & 10 & 0 & 12 & 12 & 72 & 74.7 (6.9) & $0.22(0.05)$ & $3.8(1.8)$ \\
\hline II $0.26(1.0)$ & 82 & 94 & 93 & 67 & 100 & 0 & 88 & 6 & 0 & 6 & 6 & 89 & $74.9(4.2)$ & $0.26(0.04)$ & $5.3(1.5)$ \\
\hline II $0.26(0.8)$ & 17 & 49 & 51 & 43 & 84 & 5 & 58 & 10 & 4 & 11 & 11 & 45 & $73.8(13.1)$ & $0.16(0.04)$ & $2.0(0.9)$ \\
\hline
\end{tabular}

Polar overdominance (POD) QTL were simulated based on different allele frequencies, magnitudes, and number of $\mathrm{F}_{2}$ progeny in two mating designs I: $2 \mathrm{~F}_{0}$ sires, $10 \mathrm{~F}_{0}$ dams, 8 $\mathrm{F}_{1}$ sires for 512 or $1,024 \mathrm{~F}_{2}$ progeny; II: $20 \mathrm{~F}_{0}$ sires, $80 \mathrm{~F}_{0}$ dams, $19 \mathrm{~F}_{1}$ sires for 513 or $1,026 \mathrm{~F}_{2}$ progeny. A biallelic QTL was simulated at $75 \mathrm{cM}$ for a $100 \mathrm{cM}$ chromosome with 11 equidistant markers. Each marker had four alleles with different allele frequencies in parental breeds $(0.6(0.1), 0.2(0.1), 0.1(0.2), 0.1(0.6)$ in breed A (B)). A total of 500 replicates were generated per each parameter set.

${ }^{\text {a }}$ POD I or II refer to QTL, for which differential phenotype is observed for QTL genotype 12 (I) against genotype effects of 11, 21, and 22, or genotype 21 (II) against 11, 12, and 22. POD QTL effects $(d), 0.65,0.41$, and 0.26 were defined as large, medium or small QTL, such that the QTL explained $32 \%, 12.5 \%$ or $5.1 \%$, respectively, of the phenotypic variance. Alternate QTL alleles were homogenously (1.0/0.0) or differently $(0.8 / 0.2)$ distributed in $\mathrm{F}_{0}$ parental breeds.

${ }^{b}$ Proportion of replicates in which a POD QTL was detected at a 5\% chromosome-wise level in Mendelian, paternal expression, maternal expression, POD I, or POD II models.

${ }^{c}$ If a POD QTL was detected at a 5\% chromosome-wise level in its respective POD model (Test1), a series of POD tests (Tests 2 and 3) were performed for the POD QTL to be declared as POD I, POD II, or both types.

${ }^{\mathrm{d}}$ POD QTL were tested with a series of tests to differentiate parent-of-origin effects (paternal, maternal, or partial expression) from Mendelian effects, according to THOMSEN et al. (2004).

${ }^{\mathrm{e}}$ Mean estimates (standard deviations) of the most likely QTL position from the replicates with POD QTL evidence in respective POD models.

${ }^{\mathrm{f}}$ Mean estimates (standard deviations) of POD effect $(d)$ at the most likely QTL position from the replicates with POD QTL evidence in respective POD models.

${ }^{\mathrm{g}}$ Mean estimates (standard deviations) of proportion of phenotypic variance due to POD QTL $\left(=0.75 d^{2}\right)$ from the replicates with POD QTL evidence in respective POD models. 
POD QTL were also tested for classification as nonPOD inheritance types, i.e. Mendelian, paternal or maternal expression (Table 2). Most POD QTL were declared to be partially expressed for large or medium QTL when the frequency of alternate alleles in parental breeds was equal. When the QTL size was small, or alternate alleles were segregating in parental breeds, the proportion of POD QTL declared to be partially expressed QTL was substantially decreased. Instead, the proportion of POD QTL declared as paternally or maternally expressed QTL increased, up to around $35 \%$ for small QTL when alternate alleles were fixed in parental breeds. These POD QTL that were declared as parental origin expression QTL had a similar proportion of paternally and maternally expressed QTL across QTL sizes, POD QTL types (I, II) and mating designs (Table 2). With a larger number of $\mathrm{F}_{2} \mathrm{~s}$, the proportion of POD QTL declared as partially expressed increased, up to $32 \%$ (57\% to $89 \%$ for small type II POD QTL in design II). Less than $10 \%$ of POD QTL were declared as Mendelian expressed QTL across all QTL sizes and types in both designs (Table 2).

\section{Estimates of POD QTL position and effect}

Position estimates for POD QTL that were detected in their POD models were close to unbiased and had high precision (low standard deviation) when power of QTL detection was high, i.e. for the large or medium QTL when alternate alleles were fixed in the parental breeds. When the QTL effect was small and alleles were segregating in the parental breeds, accuracy and precision of position estimates was the lowest (Table 2). Increasing sample size (number of $\mathrm{F}_{2} \mathrm{~s}$ ) gave more accurate and precise position estimates for a given QTL size and allele frequencies, which was consistent with power of detecting QTL.

Estimates of POD QTL effects were also unbiased and precise for large and medium QTL with alleles fixed for alternate alleles in the parental breeds. However, when alleles were not fixed in the grand-parents, estimates of QTL effects were downward biased and had lower precision (larger standard deviation). Generally these estimates were obtained as a function of the absolute difference between the frequencies of alternate alleles in the $F_{0}$ sire and dam breeds, i.e. $|0.8-0.2|=0.6$, such that expected POD effects were $0.39,0.25$, and 0.16 for large, medium and small POD QTL, respectively. A larger number of $F_{2}$ progeny gave more accurate and precise estimates of QTL effects, especially for small QTL with segregating alleles in the parental breeds (Table 2). Estimates of the proportion of phenotype variance explained by POD QTL were generally unbiased for large and medium QTL, slightly upward biased for small QTL, or downward biased for QTL with segregating alleles in the grand parents.

\section{Declaration of non-POD QTL as POD QTL}

Table 3 presents power to detect non-POD QTL under different genetic models, declared POD types in different mapping designs, QTL sizes, and different allele frequencies in the grand parental breeds. The power to detect QTL in the POD models was the greatest for paternal or maternal expression QTL (close to $100 \%$ for large and medium effect QTL with fixation of alternate alleles in the grand sire and dam parents), followed by complete dominance QTL, and the lowest for additive QTL. When alternate alleles were segregating in the grandparents, the power to detect QTL in the POD models decreased for all non-POD QTL, except for additive QTL, for which there was a slight increase in power compared to having homogeneous distributions of alternate alleles in the parental breeds (Table 3).

When the series of POD tests (Tests 2 and 3) were applied, most of the non-POD QTL with large or medium effects (close to $100 \%$ ) were not classified as POD under the assumption of fixed alleles in the grand parents. The accuracy of non-POD declaration was the highest for Mendelian QTL across all QTL sizes and mating designs, but the lowest for small imprinted QTL, e.g. 24\% of maternally expressed QTL were declared to be POD in design I when alternate alleles were fixed in the parental breeds. Spurious POD declaration for imprinted QTL was higher in design I than in design II, especially for small QTL. When the number of $\mathrm{F}_{2}$ progeny increased, the proportion of false POD declaration for imprinted QTL decreased, which was more pronounced in design I than in design II, when QTL size was not small, or when alternate alleles were fixed in the parental lines. For example, there was a $19 \%$ and $22 \%$ increase of non-POD declaration for paternally and maternally expressed small QTL, respectively, when parental breeds were fixed for alternate alleles in design I (Table 3).

\section{DISCUSSION}

\section{Detection of polar overdominance QTL}

A least squares regression interval mapping model was presented to detect QTL with a unique mode of gene expression, $\mathrm{POD}$, in $\mathrm{F}_{2}$ crosses between outbred lines, and a series of tests that differentiate POD QTL from Mendelian or complete imprinting QTL were developed. Our simulation results showed that, in general, POD QTL were well detected and characterized when QTL alleles were fixed for alternate alleles in the parental breeds (more than 90\% (80\%) for medium (small) QTL in $\mathrm{F}_{2}$ mating designs that have been used in pig populations). However, when the QTL effect was small and QTL alleles segregated in the parental lines, the proposed model and the series of tests did not enable effective POD QTL detection, i.e. POD 
Table 3. Power to detect Mendelian or parent-of-origin QTL under different genetic models, and proportion of the non- polar overdominance (POD) QTL declared as POD type based on simulated data

\begin{tabular}{|c|c|c|c|c|c|c|c|c|c|c|c|c|c|c|c|c|c|c|c|}
\hline \multirow{2}{*}{$\begin{array}{l}\text { QTL effect } \\
\quad \begin{array}{l}\text { (allele } \\
\text { frequency) }^{\mathrm{a}}\end{array}\end{array}$} & \multicolumn{5}{|c|}{$\begin{array}{c}\text { Power to detect QTL } \\
(\%)^{\mathrm{b}}\end{array}$} & \multicolumn{4}{|c|}{$\begin{array}{l}\text { Declared POD type } \\
(\%)^{\mathrm{c}}\end{array}$} & \multirow{2}{*}{$\begin{array}{l}\text { QTL effect } \\
\quad(\text { allele } \\
\text { frequency) }\end{array}$} & \multicolumn{5}{|c|}{$\begin{array}{l}\text { Power to detect QTL } \\
(\%)^{\mathrm{b}}\end{array}$} & \multicolumn{4}{|c|}{$\begin{array}{l}\text { Declared POD type } \\
(\%)^{\mathrm{c}}\end{array}$} \\
\hline & Mend & $\begin{array}{l}\text { Pat } \\
\text { Expr }\end{array}$ & $\begin{array}{l}\text { Mat } \\
\text { Expr }\end{array}$ & $\begin{array}{c}\text { Pod } \\
\text { I }\end{array}$ & $\begin{array}{c}\text { Pod } \\
\text { II }\end{array}$ & I & II & Both & None & & Mend & $\begin{array}{l}\text { Pat } \\
\text { Exp }\end{array}$ & $\begin{array}{l}\text { Mat } \\
\text { Exp }\end{array}$ & $\begin{array}{c}\text { Pod } \\
\text { I }\end{array}$ & $\begin{array}{c}\text { Pod } \\
\text { II }\end{array}$ & I & II & Both & None \\
\hline \multicolumn{20}{|l|}{ Design I $(\mathrm{N}=512)$} \\
\hline Pat $0.57(1.0)$ & 100 & 100 & 4 & 100 & 100 & 0 & 0 & 0 & 100 & Add $0.80(1.0)$ & 100 & 100 & 100 & 5 & 3 & 0 & 0 & 0 & 100 \\
\hline Pat $0.57(0.8)$ & 84 & 90 & 6 & 83 & 82 & 3 & 3 & 1 & 94 & Add $0.80(0.8)$ & 91 & 85 & 89 & 16 & 13 & 3 & 2 & 0 & 95 \\
\hline Pat $0.35(1.0)$ & 100 & 100 & 5 & 98 & 98 & 1 & 2 & 0 & 97 & Add 0.50 (1.0) & 100 & 100 & 100 & 6 & 4 & 1 & 0 & 0 & 99 \\
\hline Pat $0.35(0.8)$ & 65 & 84 & 5 & 64 & 60 & 8 & 6 & 2 & 84 & Add $0.50(0.8)$ & 82 & 71 & 74 & 10 & 9 & 2 & 2 & 0 & 96 \\
\hline Pat $0.23(1.0)$ & 78 & 99 & 4 & 65 & 64 & 10 & 9 & 2 & 79 & Add $0.32(1.0)$ & 98 & 87 & 88 & 6 & 6 & 1 & 2 & 0 & 97 \\
\hline Pat $0.23(0.8)$ & 32 & 68 & 4 & 39 & 36 & 11 & 10 & 2 & 77 & Add $0.32(0.8)$ & 57 & 47 & 43 & 8 & 7 & 3 & 3 & 0 & 94 \\
\hline Mat $0.57(1.0)$ & 100 & 3 & 100 & 100 & 100 & 0 & 0 & 0 & 100 & Dom 0.65 (1.0) & 100 & 100 & 100 & 89 & 85 & 0 & 0 & 0 & 100 \\
\hline Mat $0.57(0.8)$ & 86 & 5 & 93 & 84 & 84 & 4 & 2 & 0 & 93 & Dom $0.65(0.8)$ & 92 & 82 & 82 & 39 & 37 & 2 & 1 & 0 & 97 \\
\hline Mat $0.35(1.0)$ & 100 & 4 & 100 & 98 & 98 & 1 & 1 & 0 & 98 & Dom $0.41(1.0)$ & 100 & 98 & 97 & 42 & 45 & 1 & 1 & 0 & 98 \\
\hline Mat $0.35(0.8)$ & 64 & 4 & 82 & 58 & 57 & 7 & 6 & 1 & 85 & Dom $0.41(0.8)$ & 76 & 59 & 54 & 17 & 12 & 3 & 2 & 0 & 94 \\
\hline Mat $0.23(1.0)$ & 72 & 5 & 99 & 68 & 64 & 11 & 10 & 3 & 76 & Dom $0.26(1.0)$ & 99 & 65 & 67 & 18 & 19 & 2 & 1 & 0 & 97 \\
\hline Mat $0.23(0.8)$ & 34 & 4 & 64 & 30 & 31 & 9 & 7 & 2 & 82 & Dom $0.26(0.8)$ & 48 & 33 & 27 & 9 & 7 & 3 & 2 & 0 & 94 \\
\hline \multicolumn{20}{|c|}{ Design $\mathrm{I}(\mathrm{N}=1,024)$} \\
\hline Pat $0.35(1.0)$ & 100 & 100 & 5 & 100 & 100 & 0 & 0 & 0 & 100 & Add $0.50(1.0)$ & 100 & 100 & 100 & 4 & 5 & 0 & 0 & 0 & 100 \\
\hline Pat $0.35(0.8)$ & 81 & 91 & 4 & 76 & 77 & 2 & 3 & 0 & 95 & Add $0.50(0.8)$ & 92 & 84 & 85 & 9 & 10 & 1 & 1 & 0 & 98 \\
\hline Pat $0.23(1.0)$ & 97 & 100 & 5 & 91 & 94 & 1 & 1 & 0 & 98 & Add 0.32 (1.0) & 100 & 99 & 99 & 6 & 6 & 1 & 1 & 0 & 99 \\
\hline Pat $0.23(0.8)$ & 62 & 80 & 5 & 53 & 49 & 7 & 5 & 2 & 86 & Add $0.32(0.8)$ & 78 & 66 & 66 & 6 & 11 & 1 & 3 & 0 & 95 \\
\hline Mat $0.35(1.0)$ & 100 & 4 & 100 & 100 & 100 & 0 & 0 & 0 & 100 & Dom $0.41(1.0)$ & 100 & 100 & 100 & 76 & 72 & 0 & 0 & 0 & 100 \\
\hline Mat $0.35(0.8)$ & 83 & 7 & 93 & 77 & 79 & 3 & 3 & 1 & 93 & Dom $0.41(0.8)$ & 87 & 76 & 75 & 24 & 25 & 2 & 2 & 0 & 96 \\
\hline Mat $0.23(1.0)$ & 99 & 6 & 100 & 94 & 95 & 1 & 1 & 0 & 98 & Dom $0.26(1.0)$ & 100 & 94 & 90 & 30 & 37 & 1 & 0 & 0 & 98 \\
\hline Mat $0.23(0.8)$ & 58 & 7 & 82 & 52 & 57 & 5 & 5 & 2 & 88 & Dom $0.26(0.8)$ & 69 & 47 & 46 & 13 & 14 & 3 & 2 & 0 & 95 \\
\hline \multicolumn{20}{|c|}{ Design II $(\mathrm{N}=513)$} \\
\hline Pat $0.57(1.0)$ & 100 & 100 & 5 & 100 & 100 & 0 & 0 & 0 & 100 & dd $0.80(1.0)$ & 100 & 100 & 100 & 4 & 3 & 0 & 0 & 0 & 100 \\
\hline Pat $0.57(0.8)$ & 92 & 99 & 6 & 92 & 92 & 3 & 3 & 0 & 95 & Add $0.80(0.8)$ & 100 & 96 & 99 & 8 & 9 & 0 & 0 & 0 & 99 \\
\hline Pat $0.35(1.0)$ & 99 & 100 & 4 & 98 & 98 & 0 & 0 & 0 & 99 & Add $0.50(1.0)$ & 100 & 100 & 100 & 5 & 5 & 0 & 1 & 0 & 99 \\
\hline Pat $0.35(0.8)$ & 64 & 92 & 5 & 61 & 65 & 6 & 8 & 2 & 84 & Add $0.50(0.8)$ & 91 & 74 & 76 & 6 & 6 & 1 & 1 & 0 & 97 \\
\hline Pat $0.23(1.0)$ & 90 & 100 & 4 & 80 & 82 & 4 & 4 & 0 & 92 & Add 0.32 (1.0) & 97 & 82 & 86 & 4 & 4 & 1 & 1 & 0 & 97 \\
\hline Pat $0.23(0.8)$ & 42 & 80 & 7 & 41 & 39 & 9 & 8 & 2 & 82 & Add $0.32(0.8)$ & 59 & 43 & 41 & 9 & 5 & 4 & 2 & 0 & 94 \\
\hline Mat $0.57(1.0)$ & 100 & 5 & 100 & 100 & 100 & 0 & 0 & 0 & 100 & Dom 0.65 (1.0) & 100 & 100 & 100 & 87 & 88 & 0 & 0 & 0 & 100 \\
\hline Mat $0.57(0.8)$ & 95 & 4 & 99 & 92 & 93 & 1 & 2 & 0 & 97 & Dom $0.65(0.8)$ & 98 & 89 & 89 & 25 & 21 & 1 & 1 & 0 & 98 \\
\hline Mat $0.35(1.0)$ & 100 & 4 & 100 & 98 & 98 & 1 & 1 & 0 & 98 & Dom $0.41(1.0)$ & 100 & 98 & 99 & 43 & 38 & 0 & 1 & 0 & 99 \\
\hline Mat $0.35(0.8)$ & 64 & 5 & 95 & 64 & 64 & 7 & 9 & 2 & 82 & Dom $0.41(0.8)$ & 84 & 56 & 64 & 11 & 12 & 1 & 3 & 0 & 96 \\
\hline Mat $0.23(1.0)$ & 88 & 6 & 100 & 82 & 86 & 4 & 5 & 1 & 91 & Dom $0.26(1.0)$ & 98 & 69 & 65 & 19 & 19 & 1 & 3 & 0 & 96 \\
\hline Mat $0.23(0.8)$ & 39 & 4 & 81 & 38 & 35 & 9 & 10 & 3 & 78 & Dom $0.26(0.8)$ & 48 & 26 & 31 & 7 & 6 & 3 & 2 & 0 & 95 \\
\hline \multicolumn{20}{|c|}{ Design II $(\mathrm{N}=1,026)$} \\
\hline Pat $0.35(1.0)$ & 100 & 100 & 5 & 100 & 100 & 0 & 0 & 0 & 100 & Add $0.50(1.0)$ & 100 & 100 & 100 & 4 & 4 & 0 & 0 & 0 & 100 \\
\hline Pat $0.35(0.8)$ & 89 & 99 & 4 & 86 & 86 & 2 & 4 & 0 & 93 & Add $0.50(0.8)$ & 100 & 100 & 100 & 6 & 4 & 0 & 0 & 0 & 100 \\
\hline Pat $0.23(1.0)$ & 98 & 100 & 4 & 96 & 95 & 1 & 1 & 0 & 98 & Add 0.32 (1.0) & 100 & 100 & 99 & 5 & 4 & 0 & 0 & 0 & 99 \\
\hline Pat $0.23(0.8)$ & 57 & 89 & 7 & 53 & 52 & 7 & 8 & 2 & 83 & Add $0.32(0.8)$ & 86 & 68 & 67 & 4 & 6 & 1 & 1 & 0 & 98 \\
\hline Mat $0.35(1.0)$ & 100 & 5 & 100 & 100 & 100 & 0 & 0 & 0 & 100 & Dom $0.41(1.0)$ & 100 & 100 & 100 & 75 & 74 & 0 & 0 & 0 & 100 \\
\hline Mat $0.35(0.8)$ & 93 & 5 & 100 & 89 & 92 & 3 & 2 & 0 & 95 & Dom $0.41(0.8)$ & 98 & 85 & 85 & 18 & 17 & 1 & 1 & 0 & 99 \\
\hline Mat $0.23(1.0)$ & 98 & 4 & 100 & 94 & 95 & 1 & 1 & 0 & 97 & Dom $0.26(1.0)$ & 100 & 93 & 93 & 35 & 31 & 0 & 1 & 0 & 99 \\
\hline Mat $0.23(0.8)$ & 59 & 4 & 91 & 53 & 49 & 11 & 6 & 1 & 82 & Dom $0.26(0.8)$ & 76 & 49 & 49 & 10 & 10 & 2 & 2 & 0 & 96 \\
\hline
\end{tabular}

Mendelian or parent-of-origin QTL were simulated based on different allele frequencies, magnitudes, and number of $\mathrm{F}_{2}$ progeny in two mating designs I and II (see Table 2). a Add: QTL with additive effect only $(d=0)$, Dom: QTL with complete dominance $(a=d)$, Pat: QTL with paternal expression, Mat: QTL with maternal expression. QTL effects for each type of inheritance were defined, in magnitude order, as large, medium or small QTL, such that the QTL explained $32 \%$, $12.5 \%$ or $5.1 \%$, respectively, of the phenotypic variance. Alternate QTL alleles were homogenously (1.0/0.0) or differently (0.8/0.2) distributed in $\mathrm{F}_{0}$ parental breeds.

${ }^{b}$ Proportion of replicates in which a type of QTL was detected at a 5\% chromosome-wise level in Mendelian, paternal expression, maternal expression, POD I, or POD II models.

${ }^{\mathrm{c}}$ If a type of QTL was detected at a 5\% chromosome-wise level in POD models (Test1), a series of POD tests (Tests 2 and 3) were performed for the QTL to be declared as POD I, POD II, or both types.

classification down to about $35 \%$ for small QTL. When a alleles in the parental lines in design II, where a larger larger number of $F_{2}$ animals were used, the proportion of number of parents were used. However, there was limited QTL declared to be POD increased substantially for small gain in the proportion of QTL declared to be POD in design QTL, e.g. up to $25 \%$ for type I POD QTL with segregating I, in which a very small number of parents were used, e.g. 
only an increase of up to $10 \%$ (from $42 \%$ to $52 \%$ ) for type II POD QTL with segregating alleles in the parental breeds (Table 2). This result could be partly explained by varying QTL sizes in realized replicates of design I. Because few animals were used as parents $\left(\mathrm{F}_{1}\right)$ and grand-parents $\left(\mathrm{F}_{0}\right)$ in design $\mathrm{I}$, the realized breed-origin allele frequency differences in $F_{1}$ and $F_{2}$ generations would be variable among the replicates, e.g. more homozygous $F_{1}$ parents than expected, even if they were generated from the parameter set of the given allele frequency difference $(0.8 / 0.2)$, as was observed in our previous simulation study (Kim et al., 2005). This could explain the standard deviation of the estimates for POD QTL effects or of the proportion of phenotype variance due to POD QTL. Estimates of the standard deviation of the QTL variance were larger in design I than in design II for POD QTL with the same effect and with alleles segregating in the parental lines (Table 2). This may prevent some portion of replicates with smaller estimates of QTL effect in design I to get through the subsequent POD tests (2 and 3 ) for POD declaration. Also, note that proportions of replicates with POD QTL detection in their respective POD models (Test 1) were lower in design I than in design II for QTL with segregating alleles in the parental breeds (Table 2).

Our simulation results strongly suggest that POD QTL be tested with the presented tests (2 and 3 ) to differentiate POD expression from parent-of-origin expression (complete imprinting). When the series of tests of classifying POD QTL were not applied, POD QTL were classified as partially expressed QTL, i.e. 100\% declaration for large QTL with fixation of alternate alleles in the grand parental breeds (Table 2). However, when the QTL effect became smaller, the proportion of POD QTL declared as partially expressed QTL decreased and more POD QTL were declared as spurious paternal or maternal expression QTL, e.g. up to $35 \%$ for small QTL. The rate of the false declaration as paternal or maternal expression could be reduced by using a larger number of $\mathrm{F}_{2}$ offspring. However, the effect of decreasing spurious declaration of complete imprinting was in the reverse direction for QTL with segregating alleles in the grand parental breeds (Table 2).

\section{Detection of spurious polar overdominance QTL}

Our simulation results show that most Mendelian QTL or complete imprinting QTL with large or medium effect were not classified as POD type QTL. When the non-POD QTL were analyzed using POD models (Test 1), most of the large or medium non-POD QTL were detected in the POD models. However, subsequent POD tests (Tests 2 and 3) did not allow the QTL to be declared as POD type QTL (Table $3)$. However, when the QTL effect was small and breedorigin alleles were segregating in the grand parental lines, some portion of the imprinted QTL were spuriously classified as POD type QTL, which was more pronounced in the design (I) with a small number of parents (e.g. $F_{1}$ sires) (Table 3). A similar concern on the use of a small number of parents was described in de Koning et al. (2002), where spurious detection of imprinted QTL for Mendelian QTL was a problem when using an extremely small number of $\mathrm{F}_{1}$ sires. Care must be taken, therefore, in the interpretation of detected POD QTL with small effects when implemented in such a mating design, which includes the majority of porcine QTL studies (Bidanel and Rothischild, 2002). To decrease false POD classification for non POD QTL, use of a larger number of $F_{2}$ offspring is a good option, as shown in our simulation results (Table 3), or alternative strategies, i.e. mating designs, or advanced testing methods may be needed.

It must be noted that detection of imprinting effects, including polar overdominance, requires segregation of marker alleles within the lines that are crossed, and is not possible in inbred line crosses. Some imprinted genes in murines and other mammals, as well as those responsible for the callipyge phenotypes in sheep, are clustered in chromosomal domains (Tycko and Morison, 2002). Cis and trans interactions among imprinted genes around the callipyge locus resulted in different meat composition and amounts of skeletal muscle between lambs with and without callipyge genotypes (Georges et al., 2003). Thus, development of advanced imprinting models taking into account epistatic relationships between closely linked genes, as well as generation of high density maps, may be needed to better identify and characterize POD QTL.

Recently, next generation sequencing technologies have enabled the development of high throughput genotyping through high density SNP chips such as the 64k Illumina Infinium assay, which has enabled genome-wide association (GWA) studies to detect QTL with high mapping resolution (Lee et al., 2012). Application of a GWA study to detect POD QTL may be feasible if the SNP genotypes of $F_{1}$ parents and $F_{2}$ individuals are known, in which case POD tests are based on each SNP with alternate SNP alleles, e.g. allele 1 and 2, without the need for genotype information on markers that flank QTL, as in this study.

\section{ACKNOWLEDGEMENTS}

This research was supported by a grant (PJ009032) from the Next Generation BioGreen 21 Program, Rural Development Administration, Republic of Korea.

\section{REFERENCES}

Bidanel, J. P. and M. Rothschild. 2002. Current status of quantitative trait locus mapping in pigs. Pig News Inf. 23:39N$53 \mathrm{~N}$.

Choi, B. H., Y.-M. Lee, M. Alam, J.-H. Lee, T. H. Kim, K.-S. Kim, 
and J.-J. Kim. 2011. Detection of Mendelian and parent-oforigin quantitative trait loci for meat quality in a cross between Korean native pig and Landrace. Asian-Aust. J. Anim. Sci. 24:1644-1650.

Cockett, N., S. Jackson, T. Shay, F. Farnir, S. Berghmans, G. D. Snowder, D. M. Nielsen, and M. Georges. 1996. Polar overdominance at the ovine callipyge locus. Science 273:236238.

de Koning, D. J., A. P. Rattink, B. Harlizius, J. A. M. van Arendonk, E. W. Brascamp, and M. A. M. Groenen. 2000. Genome-wide scan for body composition in pigs revealed important role of imprinting. Porc. Natl. Acad. Sci. USA 97:7947-7950.

de Koning, D. J., H. Bovenhuis, and J. A. M. van Arendonk. 2002. On the detection of imprinted quantitative trait loci in experimental crosses of outbred species. Genetics 161:931-938.

Freking, B. A., J. W. Keele, M. K. Nielsen, and K. A. Leymaster. 1998. Evaluation of the ovine callipyge locus: II. Genotypic effects on growth, slaughter and carcass traits. J. Anim. Sci. 76:2549-2559.

Georges, M., C. Charlier, and N. Cockett. 2003. The callipyge locus: evidence for the trans interaction of reciprocally imprinted genes. Trends Genet. 19:248-252.

Haley, C. S., S. A. Knott, and J.-M. Elsen. 1994. Mapping quantitative trait loci in crosses between outbred lines using least squares. Genetics 136:1195-1207.

Jeon, J. T., O. Carlborg, A. Toernsten, E. Giuffra, V. Amarger, P. Chardon, L. Anderson-Eklund, K. Andersson, I. Hansson, K. Lundstroem, and L. Anderssson. 1999. A paternally expressed QTL affecting skeletal and cardiac muscle mass in pigs maps to the IGF2 locus. Nat. Genet. 21:157-158.

Kim, K.-S., J.-J. Kim, J. C. M. Dekkers, and M. F. Rothschild. 2004. Polar overdominant inheritance of a DLK1 polymorphism is associated with growth and fatness in pigs. Mamm. Genome 15:552-559.

Kim, J.-J., H. Zhao, H. Thomsen, M. F. Rothschild, and J. C. M. Dekkers. 2005. Combined line-cross and half-sib QTL analysis of crosses between outbred lines. Genet. Res. 85:235-248.
Knott, S. A., L. Marklund, C. S. Haley, K. Andersson, W. Davies, H. Ellegren, M. Fredholm, I. Hansson, B. Hoyheim, K. Lundstrom, M. Moller, and L. Andersson. 1998. Multiple marker mapping of quantitative trait loci in a cross between outbred wild boar and Large White pigs. Genetics 149:10691080.

Lee, K.-T., Y.-M. Lee, M. Alam, B. H. Choi, M. R. Park, K.-S. Kim, T.-H. Kim, and J.-J. Kim. 2012. A whole genome association study on meat quality traits using high density SNP chips in a cross between Korean native pig and Landrace. Asian-Aust. J. Anim. Sci. 25:1529-1539.

Li, Y., B. H. Choi, Y.-M. Lee, M. Alam, J.-H. Lee, K.-S. Kim, K.H. Baek, and J.-J. Kim. 2011. Characterization of QTL for growth and meat quality in combined pig QTL populations. Asian-Aust. J. Anim. Sci. 24:1651-1659.

Malek, M., J. C. M. Dekkers, H. K. Lee, T. J. Bass, and M. F. Rothschild. 2001. A molecular genome scan analysis to identify chromosomal regions influencing economic traits in the pig. I. Growth and body composition. Mamm. Genome 12:630-636.

Nezer, C., L. Moreau, B. Brouwers, W. Coppieters, J. Detilleux, R. Hanset, L. Karim, A. Kvasz, P. LeRoy, and M. Georges.1999. An imprinted QTL with major effect on muscle mass and fat deposition maps to the IGF2 locus in pigs. Nat. Genet. 21:155156

Ruckert, C., P. Stratz, S. Preuss, and J. Bennewitz. 2012. Mapping quantitative trait loci for metabolic and cytological fatness traits of connected $F_{2}$ crosses in pigs. J. Anim. Sci. 90:399-409.

Thomsen, H., H. K. Lee, M. F. Rothschild, M. Malek, and J. C. M. Dekkers. 2004. Characterization of quantitative trait loci for growth and meat quality in a cross between commercial breeds of swine. J. Anim. Sci. 82:2213-2228.

Tycko, B. and I. M. Morison. 2002. Physiological functions of imprinted genes. J. Cell. Physiol. 192:245-258.

Wilkins, J. F. and D. Haig. 2003. What good is genomic imprinting: the function of parent-specific gene expression. Nat. Rev. Genet. 4:359-368. 